\title{
Integration of mental health into primary healthcare: perceptions of stakeholders in Pakistan
}

Syed S. Hussain ${ }^{1}$, Murad Khan ${ }^{2}$, Raisa Gul ${ }^{3}$ and Nargis Asad ${ }^{2}$

${ }^{1}$ The Aga Khan University, Karachi, Pakistan (Correspondence to: Syed S. Hussain: shaz.aku@gmail.com). ${ }^{2}$ Department of Psychiatry, The Aga Khan University, Karachi, Pakistan. ${ }^{3}$ School of Nursing \& Midwifery, The Aga Khan University, Karachi, Pakistan.

\begin{abstract}
Background: In Pakistan, there is high prevalence of mental health disorders, but mental health services to address these are not well developed. To provide effective mental health services, the World Health Organization emphasizes the integration of mental health into primary health care (PHC).

Objectives: This study aimed to assess the views of key stakeholders about integration of mental health into PHC in Karachi, Pakistan.

Methods: A qualitative, exploratory study was conducted between June and September 2013 among 15 decision-making (from the Department of Health) and implementation-level stakeholders (mental health and public health professionals and primary care staff) from both the public and private sectors. Face-to-face, in-depth interviews were conducted using a semi-structured interview guide. Data were collected until theoretical saturation was achieved and conventional content analysis was carried out.

Results: Although there was general support among all the stakeholders for integration of mental health services within PHC, there were also a number of reservations. First was the perceived lack of support within the system in terms of resource allocation and acceptance from the community. Second was the lack of human resources in the field of mental health. In addition, resistance at the PHC level is likely as staff are already burdened with other preventive care services.

Conclusions: The study suggests that strong political commitment, adequate human and financial resources, and strong advocacy are needed for the integration of mental health into PHC in Pakistan.

Keywords: Mental Health Integration, Mental Health in Pakistan, Mental Health in Primary Care

Citation: Hussain SS; Khan M; Gul R; Asad N. Integration of mental health into primary healthcare: perceptions of stakeholders in Pakistan. East Mediterr Health J. 2018;24(2):146-153. https://doi.org/10.26719/2018.24.2.146.

Received: 11/05/16; accepted: 16/02/17

Copyright (c) World Health Organization (WHO) 2018. Some rights reserved. This work is available under the CC BY-NC-SA 3.0 IGO license (https:// creativecommons.org/licenses/by-nc-sa/3.o/igo).
\end{abstract}

\section{Introduction}

Mental health is an important and central component of health and the increasing prevalence of mental disorders is a burden on public health globally. Approximately $14 \%$ of the global disease burden is attributed to mental disorders $(1,2)$. Worldwide it is estimated that around 450 million people suffer from mental disorders, with around $25 \%$ of people experiencing some type of mental ill health in their lifetime $(3,4)$.

Pakistan is a South Asian developing country with a majority Muslim population and a current estimated population of about 200 million. The country faces a number of health challenges both for communicable and noncommunicable diseases (3). The country does not have a strong publicly funded primary health care (PHC) system and most people (as high as 90\%) seek health care in the highly unregulated private sector, paying out-ofpocket (3).

In the absence of a PHC or a referral system, patients access specialists directly, even for minor ailments. Other health care providers that patients commonly consult include: medically qualified general practitioners, religious healers (maulvi, pir, fakir), practitioners of hikmat (unanai medicine), homeopaths and faith healers (aamil sanyasi).

Help-seeking behaviour in Pakistan is determined by a variety of factors including perception of illness, cost, quality of care and access to health facilities. However, cost is one of the major factors determining help-seeking behaviour, particularly as most health care is out-ofpocket expenditure.

There is a high prevalence of common mental disorders in Pakistan, ranging from a low of $25 \%$ (urban areas) to a high of $72 \%$ (rural areas) for females and between 10\% (urban) and 44\% (rural) for males (5). A study in Karachi (Pakistan's largest city and main commercial centre) reported that $17 \%$ of the respondents had "psychological distress" (6). These figures are higher than other developing countries with similar socioeconomic indicators $(5,7-10)$.

Factors associated with high rates of common mental disorders include high levels of social adversity experienced by the population, poor housing and low education. For women, additional factors include: their status in a strongly patriarchal society, marital status (being married), early age at marriage, number of children, economic dependence on the husband, joint and 
extended family systems and domestic violence $(10,11)$.

The above-mentioned prevalence figures should be viewed critically from the transcultural psychiatry perspective. There is currently considerable debate on the universality of mental disorders and whether notions of mental disorders, which are largely based on Western culture, can be simply transposed to nonWestern cultures (12). The effect of applying Western ideas of mental illnesses to non-Western settings is to overlook the varied and unique experiences of distress of people in many low- and middle-income countries that are shaped by culture, traditions, disease attribution, help-seeking behaviour as well as sociopolitical and religious factors (13). Indeed, "social suffering" may be a more appropriate concept to explain the distress of living under extremely adverse social conditions, as opposed to the Western notion of psychopathology (13). This has serious implications for epidemiological research as well as for programmes for mental health.

Mental health services are poorly developed in Pakistan. This is not only due to poor resource allocation but also to a general ignorance of issues related to mental health at all levels of government planning and implementation. Mental health does not have a separate budget but is believed to be about $1 \%$ of the national health budget, which itself is about $0.8 \%$ of the gross national product. The psychiatrist to population ratio is about 1 psychiatrist to $0.5-1$ million people. The number of psychologists is similarly low. There are no psychiatric social workers. Most psychiatrists are located in large urban centres, while the majority of the population lives in the rural areas where mental health services are all but non-existent (14). A total of 3729 mental health facilities are present in the country, $46 \%$ of which are supposed to provide community-based mental health services $(15,16)$. There are 5 mental health hospitals in the country, with the ratio of 1.9 beds:100 000 population (15). These facilities are mostly located in cities.

Although psychiatry and behavioural sciences are now being taught in many medical schools in Pakistan, psychiatry is examined as a certifying exam in only 1 medical school (17). The result is that generations of Pakistani doctors have passed through medical training with little or no exposure to issues related to mental health (18).

The national mental health policy of 2003 advocated for a mental health component in PHC as well as development of community-based mental health services (14), but this has never been implemented. In a large number of cases mental disorders remain undiagnosed. A study of predictors of treatment delay for depressive disorders in Pakistan showed a mean delay of 4.5 years from the onset of the first episode of depression to the first contact with a psychiatrist (19). A recent study estimated the economic burden of mental illnesses in Pakistan as US\$ 4.2 billion annually, with medical care costs and productivity losses contributing $37 \%$ and $59 \%$ to the total costs respectively (3). This study also estimated that, assuming a gate-keeping role of PHC, there could be a saving of US\$ 1.6 billion in the total costs (3).

In a number of low- and middle-income countries, e.g. the Islamic Republic of Iran, India, Uganda and Brazil, mental health services have been integrated in PHC and their integration has been shown to be cost-effective, improve access to care and reduce social stigma and discrimination (20-22).

Hence to provide accessible mental health care, it is important to integrate mental health into PHC (15). The role of stakeholders, including decision-makers, in implementation is crucial. In some studies, their views have been identified as barriers to integration (23). Therefore, exploration of their views about integration is important. In this study, we explored the perceptions of stakeholders about the integration of mental health into PHC and the perceived barriers to this. We also explored their views on resources required for the integration process.

\section{Methods}

\section{Study design}

A qualitative, exploratory design was used for the study. Qualitative studies require the maintenance of trustworthiness and to do so in this study, the criteria of Lincoln and Guba (1989) were followed which include credibility, transferability and conformability. For credibility, an interview guide was developed in consultation with the study supervisor and research project committee members. Planned and unplanned probes were added to the interview guide after piloting it on 2 participants. In-depth interviews were conducted to obtain detailed information on phenomena. To maintain conformability of the study, the analysis was performed under the direction of the supervisor and research project committee members to ensure the quality of the findings. For transferability, the research process was presented in the form of a thesis, which included all the details about the research methodology.

\section{Study setting}

The study was conducted between June and September 2013 in Karachi, Pakistan's largest city and its main commercial and business centre. Decision-making and implementation level stakeholders were interviewed for the study. These personnel were also more accessible than the more senior officials such as the minister or secretary of health. Also, although patients and service users are also important stakeholders in the development of any health service, they were not included in this study as the focus was on decision-makers and policy implementers.

\section{Study participants}

The decision-level stakeholders were individuals from the Department of Health (who are involved in decisionmaking for health services) of Sindh province (one of the 4 provinces of the country). Implementation-level stakeholders were mental health and public health 
professionals and primary care staff. The participants were selected from both public and private health institutions. The inclusion criteria for selection were:

- Health care professionals in the field of mental health and/or public health with a minimum of 5 years' experience in the field

- Primary care physicians with more than 2 years of experience

- Officials from the Department of Health (provincial) involved in making policy decisions for mental health.

\section{Sampling and data collection}

Participants who met the inclusion criteria were contacted by email and telephone to assess their willingness to participate. A purposive sampling strategy was used to recruit study participants (24). Participants were interviewed at their place of work. Face-to-face indepth interviews were conducted using a semi-structured interview guide developed by the researchers, which was piloted on 2 participants. Interviews took $45-60$ minutes. Participants were given the choice to respond in English or Urdu. Data were collected until theoretical saturation (25) was achieved, which was reached after interviewing 15 participants. The primary investigator (SSH) conducted all the interviews. Each interview was recorded with the permission of the participant and later transcribed.

\section{Data analysis}

Data of the in-depth interviews were analysed using NVivo software, version 10 (QSR International). Conventional content analysis was used as no prior codes were established for analysis.

The primary investigator checked the transcribed data with the recording to verify them. The transcribed data were read several times for familiarization and the information was matched with the objectives and research questions to ensure that the collected data answered the research questions. Each interview was assigned a specific code to maintain confidentiality.

\section{Ethical considerations}

Approval for the study was sought and obtained from the Ethical Review Committee of The Aga Khan University, the Department of Health, Government of Sindh and participating institutions. Informed, written consent was taken from all the participants.

\section{Results}

\section{Characteristics of the participants}

The demographic characteristics and work background of the participants are shown in Table 1. The majority were males, with an age range of 30-39 years. Most of them had a master's degree, followed by those with a basic medical degree (MBBS) and post-graduate medical qualification (FCPS). Most of the participants had experience in public health and psychiatry and there was equal participation from both public and private sectors.

\section{Importance of mental health}

All the participants agreed that mental health was important at the individual as well as at the community, societal and national levels. They further stated that community is made up of individuals and if any individual is mentally unhealthy, it will affect the whole community and the nation at large. They pointed out that the current situation of mental health in Pakistan was very poor, given the frequent episodes of terrorism and poor law and order in the country. They indicated that there was no emphasis on preventive measures. They supported the idea that integrating mental into PHC was urgently needed as societal stress was very high and people were suffering from emotional and psychological distress. One of the participants stated: "I think mental health is extremely important at all levels. Obviously, if the individual is mentally healthy, they will contribute to building a more healthy and functional community and society."

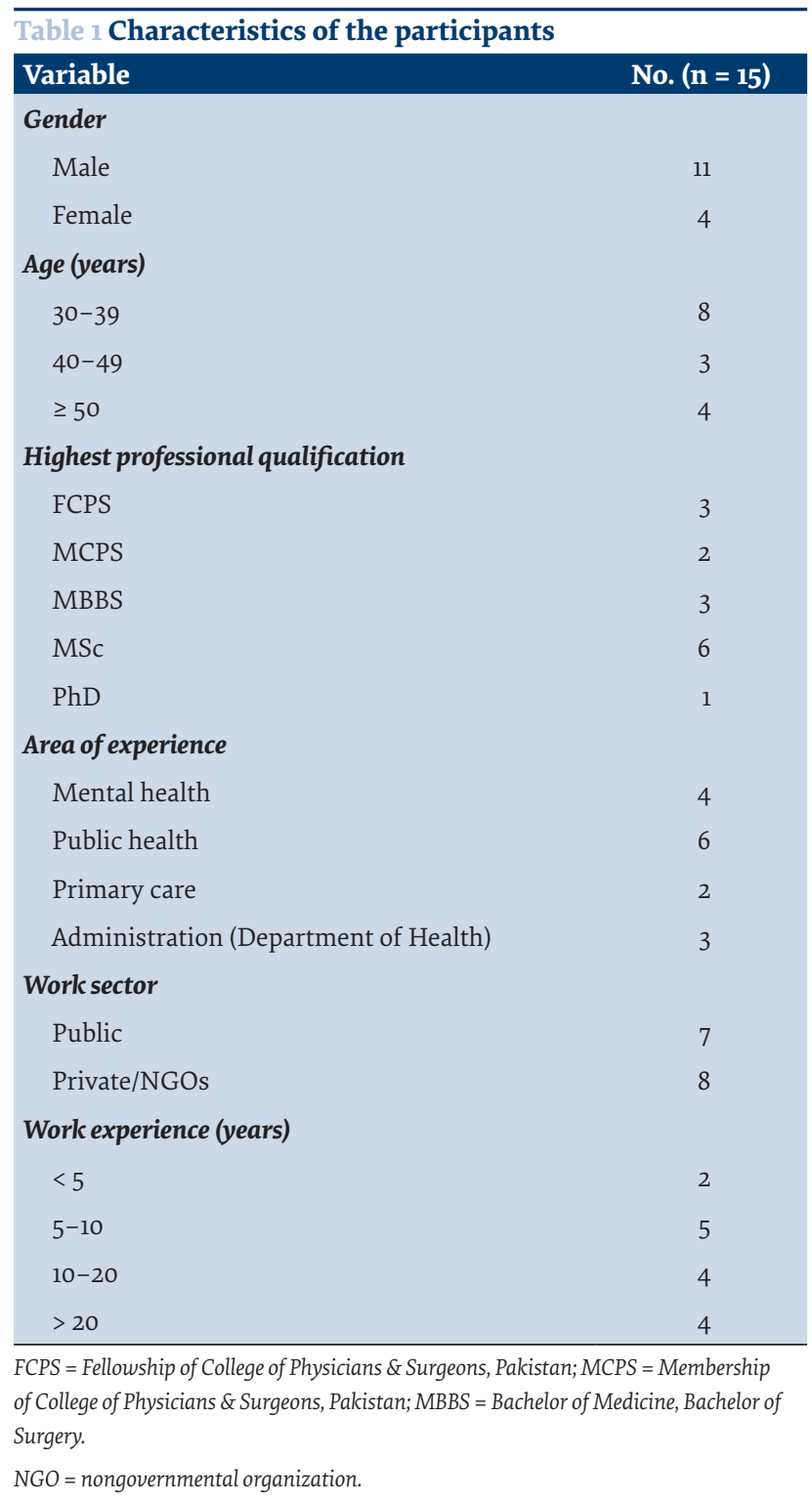




\section{Barriers to integration}

While the majority of the participants emphasized the importance of mental health and its integration in PHC, they also noted several barriers that might hinder the process of integration. The barriers were at system, implementation and community levels.

At the system level, lack of political commitment was considered a major barrier to integration. Many participants felt that as integration was a major decision, there would need to be considerable modification of the current system to facilitate the process. Such changes in the system were not possible without strong political commitment. One of the participants stated that: "So the biggest barriers are resources and the lack of political will and commitment. The day we have resources and political will and commitment, things will work out and communities will follow suit."

At the implementation level, lack of human resources was identified as a key barrier to the integration process. Participants mentioned that there was a need for adequate human resources, i.e. doctors, nurses and community workers, but that the proportion of care providers to the population in Pakistan was very low. One of the participants stated: "At the moment, the main barrier is lack of human resources."

Some participants highlighted the fact that there would be resistance at the PHC level because of the shortage of staff and medical supplies, and the lack of proper monitoring. These issues already contribute to low utilization of PHC facilities. Without addressing these fundamental issues, integration would be unlikely to be effective. In addition, there would be reluctance from staff as their workload would increase because mental health disorders generally need more time for proper diagnosis and management.

At the community level, lack of awareness among the community was considered a barrier to integration. The study participants highlighted the fact that there were many misconceptions about mental disorders, and the acceptance of people with mental health problems among the community was generally low. Some participants felt there was a lack of demand from the community for integration of mental health into PHC. Government tends to provide services to people according to their demands and if the people demand integration, the government may consider it as an option. Raising awareness was considered a key element to induce demand from the community. One of the participants stated: "For me, access is linked to the felt need and the demand from the general public. In order for the public to demand that, they need to be sensitized so if spending has to be directed towards mental health, a portion of it has to be directed in this direction as well."

\section{Resources required for integration}

Capacity building was considered an important step in the process of integration. Participants highlighted the importance of human resources for integration and proposed suggestions to improve human resources capacity in mental health. Capacity building includes the training of primary care physicians in the diagnosis, treatment and referral of common mental health conditions, particularly as exposure to psychiatry at the undergraduate level was far from satisfactory. Similarly, training of nurses and community health workers is also a key element to provide awareness and education to the community.

A number of participants highlighted the need for under- and post-graduate level training in mental health. This would familiarize physicians with mental health issues and may go a long way to addressing the lack of human resources in this area, particularly those who work at the PHC level.

Participants also said that the role of faith healers in mental health should be taken into account, as they are the first line of contact in many cases. This appeared to be deeply embedded in the Pakistani culture. By providing education to faith healers, they could be made aware of the need to refer patients who require medical/psychiatric treatment. One of the participants stated: "Human resource are obviously needed, as you already have the infrastructure of a health system all over the country. We have BHUs (basic health units) in every area, as well as RHCs (rural health centres), and DHQs (district headquarter) hospitals."

Financial resources were considered to be the major factor in the process of integration. Integration requires funding for human resources development and restructuring of the PHC system. The provision of essential psychiatric medications also requires substantial financing. For integration of mental health, the overall health budget needs to be increased and a greater proportion allocated for mental health. One of the participants stated: "And one of the most important things is the funds because allocation of the budget for health is already very low. What we spend on health is very low when compared with other developing countries."

Some participants also highlighted the importance of advocacy and more research in the field of mental health. Committed people are needed in order to advocate for mental health. Nongovernmental organizations could play a significant role in this area. A lack of research was also considered an important issue in mental health. The importance of having robust data to inform policy on which services are developed was highlighted by some participants.

\section{Discussion}

To the best of our knowledge this is the first study that explored the perceptions of stakeholders about the integration of mental health into PHC in Pakistan. The study highlighted stakeholders' perspectives on the importance of mental health, their views on barriers to integration and the resources required for integration.

A study in Uganda and South Africa showed that a common implementation framework incorporating a community collaborative multisectoral, task shifting and self-help approach to integrating mental health into 
PHC holds promise for closing the treatment gap for mental disorders in low- and middle-income countries at the district level (23). Across both country sites, self-help groups generally incorporated some form of livelihoodgenerating activities. This was important to break the vicious cycle of poverty and mental ill health, which is now well accepted (26), and promote social inclusion, which in turn can help reduce stigma and discrimination.

Bracken et al. (2016) arguing against the globalization of mental disorders and the imposition of Western models of mental illness on non-Western settings, wrote that, "the stark reality for the vast majority of people in the non-Western world, transcending everything, is poverty. Currently one quarter of the global population lives in near destitution and 3.5 million children die of starvation annually. What is 'mental health' in this broken social world?" (12). What this clearly means that it is not simply a question of integrating mental health into PHC but also of addressing some of the fundamental contextual factors (such as poverty) without which it is highly unlikely mental health issues can be alleviated.

In the present study community, system and implementation level barriers were discussed for integration of mental health into PHC. Stigma and lack of demand at the community level, low priority and financial constraints at the system level, lack of human resources and resistance within PHC at the implementation level were highlighted. Strong political commitment, channelling of additional financial resources towards health and innovative human resources management were identified for the integration of mental health into PHC. The Uganda and South Africa study on integration showed that improved mental health literacy within communities can strengthen demand for services (23). This can act as a catalyst and advocacy opportunity for increasing the public health priority afforded to mental health by governments in low- and middle-income countries and donor agencies.

In Pakistan, like other developing countries, little attention is given to mental health at the policy level. This study confirmed that mental health, especially prevention, is of little priority at the health policy-making level. Health in general and mental health in particular have always received meagre resource allocation in Pakistan. Studies on barriers to improving mental health show that it has always been low on the public health agenda at national as well as international levels, which affects funding for mental health (14,27-29). The effect of strong political commitment can be seen in Sri Lanka where before the 2004 tsunami, mental health resources were mainly focused around large cities. The political interest shown towards mental health changed dramatically after the tsunami and the government initiated a new national health policy with more resource allocation for mental health. By the end of $2007,67 \%$ of districts were providing acute inpatient care for mental health as compared to a pre-policy level of $37 \%(30)$.

A lack of human resources is a universal problem especially in low- and middle-income countries. Adequate human resources are needed to achieve better health outcomes. The issue is even more critical in the context of mental health. Unlike other health care settings, mental health management is more human than technology intensive. In low- and middle-income countries, shortage of psychiatrists, psychiatric nurses, psychologists and social workers are major impediments to treatment and care (31-33). Our study highlights the shortage of human resources for mental health in Pakistan, which are critical for integrating mental health in PHC. It suggests the need to teach mental health at undergraduate and postgraduate levels so that more graduates can take up mental health as profession. These findings are consistent with published literature (32-36).

Another barrier identified by our study was resistance by PHC staff as they believed integration would impose additional responsibilities on them. Issues of governance, inadequate supervision and monitoring of staff, and lack of professionalism were all highlighted as barriers to effective integration. Studies have shown that resistance by policy-makers and health workers hampers development of mental health services (27). Strong advocacy at the planning stage is crucial to overcome resistance and negative attitude of PHC staff (21).

The burden of mental disorders is huge and growing, and current strategies are not effective to improve the mental health care situation in Pakistan. Existing services are ineffective in terms of accessibility, quality and affordability. There are a limited number of institutions providing mental health services in Pakistan and human resources are insufficient. Furthermore, there is a lack of awareness at all levels. At the provider level, the focus is mainly on tertiary care and preventive strategies are all but missing for mental health.

The country lacks a proper mental health policy. Mental health has received very little policy attention from the very beginning. Health policy-makers are generally non-technical and lack an understanding of the needs of the population. With the current health budget, the process of integration would appear to be very difficult. However, our study shows a general willingness among stakeholders for integration of mental health into PHC, although they had strong underlying reservations.

The strength of this study is that it was conducted in an area of health that is given very low priority in the country. Data were triangulated by collecting data from stakeholders at different levels. A weakness of the study is that we did not include the perceptions of service users (patients), who are important stakeholders in any service planning. Future studies should include this important group as well.

The study explored the perceptions of stakeholders in Karachi, the capital city of Sindh province, one of the 4 provinces of Pakistan. Hence, the findings of the study cannot be generalized to other areas of the country. There is need to conduct similar studies in other provinces of Pakistan. 


\section{Conclusions}

Our findings show that mental health is considered an important component of health by both decision-makers and implementation-level stakeholders in Pakistan. The importance of mental health at individual, community and national level was emphasized. The study also showed that there are many flaws in the current mental health services in Pakistan and mental health problems are not being addressed properly. The stakeholders felt that to integrate mental health into PHC there was need for strong political commitment, human and financial resources, community level awareness and strong advocacy. The current PHC system itself would need to be strengthened before integration could be implemented. At the same time integration and development of services need to be carefully planned in accordance with the prevailing health-seeking behaviour of the local population, as well by as other demographic, cultural and socioeconomic factors, since these factors are likely to contribute significantly to their overall acceptance and effectiveness.

\section{Acknowledgements}

The participants of this study are acknowledged for their valuable time. The authors also acknowledge Mr Abdul Qayum for his feedback on the language of the manuscript.

Funding: This study was a thesis requirement for a master's degree. The cost of travel and logistics were reimbursed by the Board of Graduate Studies of The Aga Khan University.

Competing interests: None declared.

\section{Intégration de la santé mentale aux soins de santé primaires : perceptions des parties prenantes au Pakistan}

\section{Résumé}

Contexte : Au Pakistan, la prévalence des troubles de santé mentale est élevée, mais les services de santé mentale visant à prendre en charge ces troubles ne sont pas bien développés. Afin de fournir des services de santé mentale efficaces, l'Organisation mondiale de la Santé met l'accent sur l'intégration de la santé mentale dans les soins de santé primaires.

Objectifs : La présente étude visait à évaluer les points de vue des parties prenantes clés au sujet de l'intégration de la santé mentale dans les soins de santé primaires à Karachi (Pakistan).

Méthodes : Une étude exploratoire qualitative a été conduite entre juin et septembre 2013 parmi 15 parties prenantes chargées de la prise de décisions (relevant du Département de la Santé) et de la mise en œuvre (professionnels de la santé mentale et de la santé publique et équipes de soins primaires) issues des secteurs public et privé. Des entretiens approfondis en face-à-face ont été menés en recourant à un guide d'entretiens semi-structurés. Les données ont été recueillies jusqu'à saturation théorique, et une analyse de contenu conventionnelle a été réalisée.

Résultats : Bien que les parties prenantes soutiennent dans leur ensemble l'intégration de la santé mentale aux soins de santé primaires, certaines d'entre elles ont émis des réserves. La première concernait le manque de soutien perçu au sein de ce système en ce qui concerne l'allocation des ressources et l'acceptation par la communauté. Ensuite venait le manque de ressources humaines dans le domaine de la santé mentale. En outre, il est probable de rencontrer une résistance au niveau des soins de santé primaires, les équipes étant déjà surchargées par d'autres services de soins préventifs.

Conclusions : Cette étude suggère qu'un engagement politique fort, des ressources humaines et financières suffisantes et un plaidoyer énergique sont requis en vue de l’intégration de la santé mentale aux soins de santé primaires au Pakistan.

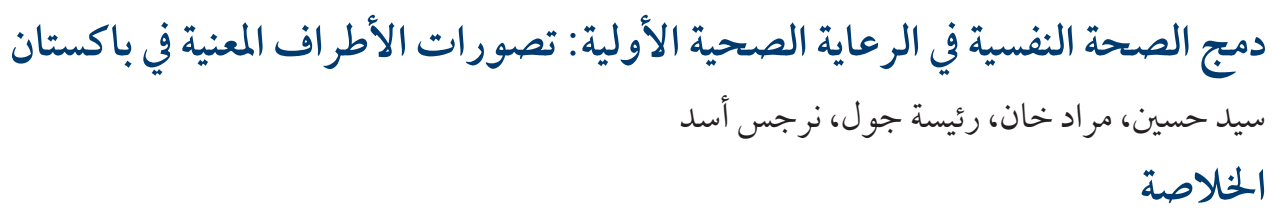




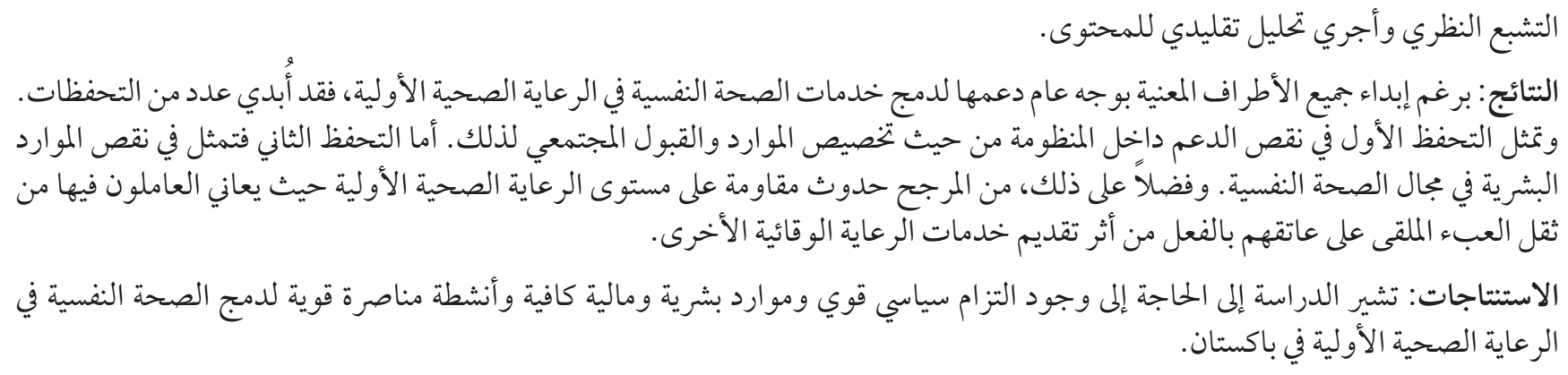

\section{References}

1. Prince M, Patel V, Saxena S, Maj M, Maselko J, Phillips MR, et al. No health without mental health. Lancet. 2007 Sep 8;370(9590):859-77. http://dx.doi.org/10.1016/S0140-6736(07)61238-0 PMID:17804063

2. Malata AA. Gap analysis for the national health research agenda of Malawi. 2010.

3. Malik MA, Khan MM. Economic burden of mental illnesses in Pakistan. J Ment Health Policy Econ. 2016 Sep;19(3):155-66. PMID:27572143

4. Preventing suicide: a global imperative. Geneva: World Health Organization; 2014 (http://apps.who.int/iris/bitstre am/10665/131056/1/9789241564779_eng.pdf, accessed 8 November 2017).

5. Mumford DB, Minhas FA, Akhtar I, Akhter S, Mubbashar MH. Stress and psychiatric disorder in urban Rawalpindi. Community survey. Br J Psychiatry. 2000 Dec;177(6):557-62. http://dx.doi.org/10.1192/bjp.177.6.557 PMID:11102332

6. Kidwai R. Demographic factors, social problems and material amenities as predictors of psychological distress: a cross-sectional study in Karachi, Pakistan. Soc Psychiatry Psychiatr Epidemiol. 2014 Jan;49(1):27-39. http://dx.doi.org/10.1007/s00127-013-0692-0 PMID:23620098

7. Ali B, Saud Anwar M, Mohammad SN, Lobo M, Midhet F, Ali SA. Psychiatric morbidity: prevalence, associated factors and significance. J Pak Med Assoc. 1993 Apr;43(4):69-70. PMID:8230655

8. Husain N, Creed F, Tomenson B. Depression and social stress in Pakistan. Psychol Med. 2000 Mar;30(2):395-402. http://dx.doi. org/10.1017/So033291700001707 PMID:10824659

9. Mumford DB, Nazir M, Jilani FU, Baig IY. Stress and psychiatric disorder in the Hindu Kush: a community survey of mountain villages in Chitral, Pakistan. Br J Psychiatry. 1996 Mar;168(3):299-307. http://dx.doi.org/10.1192/bjp.168.3.299 PMID:8833683

10. Mumford DB, Saeed K, Ahmad I, Latif S, Mubbashar MH. Stress and psychiatric disorder in rural Punjab. A community survey. Br J Psychiatry. 1997 May;170(5):473-8. http://dx.doi.org/10.1192/bjp.170.5.473 PMID:9307700

11. Qadir F, de Silva P, Prince M, Khan M. Marital satisfaction in Pakistan: A pilot investigation. Sexual Rel Ther. 2005;20.(2.):195-209.

12. Bracken P, Giller J, Summerfield D. Primum non nocere. The case for a critical approach to global mental health. Epidemiol Psychiatr Sci. 2016 Dec;25(6):506-10. http://dx.doi.org/10.1017/S2045796016000494 PMID:27522868

13. Summerfield D. Global mental health is an oxymoron and medical imperialism. 2013. BMJ. 2013 May 31;346:f3509. doi: 10.1136/ bmj.f3509 PMID: 23729293

14. Karim S, Saeed K, Rana MH, Mubbashar MH, Jenkins R. Pakistan mental health country profile. Int Rev Psychiatry. 2004 FebMay;16(1-2):83-92. http://dx.doi.org/10.1080/09540260310001635131 PMID:15276941

15. World Health Organization. WHO-AIMS report on mental health system in Pakistan. 2009 (http://www.who.int/mental_health/ pakistan_who_aims_report.pdf, accessed 8 November 2017).

16. Mubbashar MH, Saeed K. Development of mental health services in Pakistan. East Mediterr Health J. 2001 May;7(3):392-6. PMID:12690758

17. Naeem F, Ayub M. Psychiatric training in Pakistan. Med Educ Online. 2004 Dec;9(1):4369. doi: 10.3402/meo.v9i.4369 PMID: 28253115 .

18. Khan MM. Through the back door: the college and award of membership without examination. Psychiatr Bull. 2006;30(1):3-5. http://dx.doi.org/10.1192/pb.30.1.3

19. Sadruddin S. Predictors of treatment delay in depressive disorder in Pakistan [PhD thesis]. Toronto: Department of Health Policy, Management and Evaluation, University of Toronto; 2007.

20. World Health Organization. WHO-AIMS report on mental health system in The Islamic Republic of Iran. 2006. (http://www.who. int/mental_health/evidence/who_aims_report_iran.pdf, accessed 8 November 2017).

21. Integrating mental health into primary care: a global perspective. Geneva: World Health Organization and World Organization of Family Doctors (Wonca); 2008 (http://www.who.int/mental_health/policy/Mental\%2ohealth\%20+\%20primary\%20care-\%20 final\%20low-res\%20140908.pdf, accessed 8 November 2017).

22. Ovuga E, Boardman J, Wasserman D. Integrating mental health into primary health care: local initiatives from Uganda. World Psychiatry. 2007 Feb;6(1):60-1. PMID:17342230 
23. Petersen I, Ssebunnya J, Bhana A, Baillie K; MhaPP Research Programme Consortium. Lessons from case studies of integrating mental health into primary health care in South Africa and Uganda. Int J Ment Health Syst. 201104 15;5(8):8. http://dx.doi. org/10.1186/1752-4458-5-8 PMID:21496242

24. Palinkas LA, Horwitz SM, Green CA, Wisdom JP, Duan N, Hoagwood K. Purposeful sampling for qualitative data collection and analysis in mixed method implementation research. Adm Policy Ment Health. 2015 Sep;42(5):533-44. http://dx.doi.org/10.1007/ s10488-013-0528-y PMID:24193818

25. Francis JJ, Johnston M, Robertson C, Glidewell L, Entwistle V, Eccles MP, et al. What is an adequate sample size? Operationalising data saturation for theory-based interview studies. Psychol Health. 2010 Dec;25(10):1229-45. http://dx.doi. org/10.1080/08870440903194015 PMID:20204937

26. Lund C, Breen A, Flisher AJ, Kakuma R, Corrigall J, Joska JA, et al. Poverty and common mental disorders in low and middle income countries: A systematic review. Soc Sci Med. 2010 Aug;71(3):517-28. http://dx.doi.org/10.1016/j.socscimed.2010.04.027 PMID:20621748

27. Saraceno B, van Ommeren M, Batniji R, Cohen A, Gureje O, Mahoney J, et al. Barriers to improvement of mental health services in low-income and middle-income countries. Lancet. 2007 Sep 29;370(9593):1164-74. http://dx.doi.org/10.1016/So1406736(07)61263-X PMID:17804061

28. Kathol RG, Butler M, McAlpine DD, Kane RL. Barriers to physical and mental condition integrated service delivery. Psychosom Med. 2010 Jul;72(6):511-8. http://dx.doi.org/10.1097/PSY.obo13e3181e2c4ao PMID:20498293

29. Lancet Global Mental Health Group, Chisholm D, Flisher AJ, Lund C, Patel V, Saxena S, et al. Scale up services for mental disorders: a call for action. Lancet. 2007 Oct 6;370(9594):1241-52. http://dx.doi.org/10.1016/So140-6736(07)61242-2 PMID:17804059

30. Gambheera H. Mental health services in Sri Lanka. In: Bhugra D, Tse S, Ng R, Takei N, eds. Routledge handbook of psychiatry in Asia. London: Routledge: 2015:115-26.

31. Eaton J, McCay L, Semrau M, Chatterjee S, Baingana F, Araya R, et al. Scale up of services for mental health in low-income and middle-income countries. The Lancet. 2011 Oct 29;378(9802):1592-603. https://doi.org/10.1016/So140-6736(11)60891-X PMID:22008429

32. Kakuma R, Minas H, van Ginneken N, Dal Poz MR, Desiraju K, Morris JE, et al. Human resources for mental health care: current situation and strategies for action. Lancet. 2011 Nov 5;378(9803):1654-63. https://doi.org/10.1016/So140-h6736(11)61093-3 PMID:22008420

33. Saxena S, Thornicroft G, Knapp M, Whiteford H. Resources for mental health: scarcity, inequity, and inefficiency. Lancet. 2007 Sep 8;370(9590):878-89. https://doi.org/10.1016/S0140-6736(07)61239-2 PMID:17804062

34. Dale JT, Bhavsar V, Bhugra D. Undergraduate medical education of psychiatry in the West. Indian J Psychiatry. 2007 Jul;49(3):1668. http://dx.doi:10.4103/0019-5545.37315 PMID:20661380

35. Bruckner TA, Scheffler RM, Shen G, Yoon J, Chisholm D, Morris J, et al. The mental health workforce gap in low- and middle-income countries: a needs-based approach. Bull World Health Organ. 2011 Mar 1;89(3):184-94. http://dx.doi.org/10.2471/ BLT.10.082784 PMID:21379414

36. Jacob KS, Sharan P, Mirza I, Garrido-Cumbrera M, Seedat S, Mari JJ, et al. Mental health systems in countries: where are we now? Lancet. 2007 Sep 22;370(9592):1061-77. http://dx.doi.org/10.1016/So140-6736(07)61241-o PMID:17804052 